\title{
RefineNet-based automatic delineation of the clinical target volume and organs at risk for three-dimensional brachytherapy for cervical cancer
}

\author{
Xue Jiang, Fang Wang, Ying Chen, Senxiang Yan \\ Department of Radiation Oncology, the First Affiliated Hospital, Zhejiang University School of Medicine, Hangzhou, China \\ Contributions: (I) Conception and design: X Jiang; (II) Administrative support: S Yan; (III) Provision of study materials or patients: F Wang; (IV) \\ Collection and assembly of data: Y Chen; (V) Data analysis and interpretation: X Jiang; (VI) Manuscript writing: All authors; (VII) Final approval of \\ manuscript: All authors. \\ Correspondence to: Senxiang Yan. Department of Radiation Oncology, the First Affiliated Hospital, Zhejiang University School of Medicine, \#79 \\ Qingchun Road, Hangzhou 310003, China. Email: yansenxiang@zju.edu.cn.
}

Background: In recent years, high-precision image-guided intensity-modulated radiation therapy combined with three-dimensional (3D) high-dose-rate (HDR) brachytherapy (BT) has become a recommended technique for radical radiotherapy for cervical cancer. This study first employed contrastlimited adaptive histogram equalization (CLAHE) for preprocessing of input data to achieve image enhancement. In this way, rapid and accurate automatic delineation of the clinical target volume (CTV) and organs at risk (OARs) in 3D BT for cervical cancer was achieved.

Methods: Two hundred cervical cancer patients who underwent radical radiotherapy from January 2016 to December 2018 were selected. After collecting the computed tomography (CT) image data of a patient, we constructed the radiotherapy CTV and OAR image libraries. A RefineNet-based deep learning protocol was used to segment the CTV and OARs for 3D BT for cervical cancer. In this study, a total of 1,000 rounds of training were carried out, and the model with the best performance was selected for subsequent iterative tuning. Finally, the clinical test was carried out, in which the CT images of 10 cases were tested one by one. The manual delineation results and the model output results for the CTV and OARs were compared to measure the performance of the model.

Results: Compared with the manually delineated CTV, the RefineNet model-based segmented CTV had a higher Dice similarity coefficient (DSC), Hausdorff distance (HD), and overlap index (OI), which were 0.861 , 6.005, and 0.839, respectively. For OARs, the RefineNet-based model obtained the best results for bladder segmentation (DSC: $85.96 \%$ ), respectively. The mean duration of RefineNet-based automatic contour processing of the CTV was $70 \mathrm{~s}$, and the mean durations of RefineNet-based automatic delineation of the bladder, rectum, sigmoid colon, and small intestine were 67, 67.4, 63.8, and $60.8 \mathrm{~s}$, respectively. The total time saved by RefineNet was approximately $60 \%$.

Conclusions: The RefineNet-based automatic delineation model for 3D BT for cervical cancer is a stable and highly consistent automatic delineation algorithmic model that has the potential to improve the consistency of target region delineation, simplify the radiotherapy procedure, and achieve rapid and accurate automatic delineation of CTVs and OARs.

Keywords: RefineNet network; cervical cancer; three-dimensional brachytherapy (3D BT); automatic delineation

Submitted Jun 29, 2021. Accepted for publication Oct 19, 2021.

doi: $10.21037 /$ atm-21-4074

View this article at: https://dx.doi.org/10.21037/atm-21-4074 


\section{Introduction}

Cervical cancer is one of the most common malignancies in women, and locally advanced malignancies account for $70 \%$ of all cervical cancers in developing countries (1). Surgery alone cannot achieve the goal of radical treatment in most cervical cancer patients, so adjuvant radiotherapy is required, but postoperative adjuvant radiotherapy will result in more complications (2). Therefore, radical radiotherapy is the preferred treatment for cervical cancer. Brachytherapy (BT) is a necessary means of radical radiotherapy for locally advanced cervical cancer, and high-precision image-guided downregulation of intense radiotherapy combined with threedimensional high-dose-rate (3D HDR) BT has become the recommended technique of radical radiotherapy for cervical cancer. Image-guided 3D BT can directly display the location of the applicator, help to delineate, plan and evaluate the target area of radiotherapy, and enable the tumor to obtain the best dose conformability. It can not only increase the dose of the tumor target, but also minimize the radiation dose of adjacent normal tissues (3). In recent years, highprecision image-guided intensity-modulated radiation therapy combined with 3D HDR BT has become a recommended technique for radical radiotherapy of cervical cancer.

3D HDR BT can provide the best dose conformity to the tumor and minimize the radioactive dose to adjacent normal tissues. This high-precision radiotherapy technique can only be performed when high-precision image localization is used to accurately define the tumor target region. The Groupe Européen de Curiethérapie and the European Society for Radiotherapy \& Oncology (GEC-ESTRO) recommends the delineation of target regions in 3D BT for cervical cancer based on T2-weighted imaging (T2WI) magnetic resonance imaging (MRI) images and gynecological examination results (4). However, due to technical limitations, the use of MRI in guiding and planning the treatment for clinical radiotherapy is still extremely limited. At present, computed tomography (CT) images are still the most used in China for delineation of target regions in 3D BT for cervical cancer. The self-adaptive histogram equalization algorithm, i.e., contrast-limited adaptive histogram equalization (CLAHE) (5) is a computer image processing technique that increases the local contrast and clarity of an image, so it can be used to achieve accurate image identification for localization in 3D BT.

The radiologist's experiences and preferences can result in deviations in the target regions due to individual or external reasons (6-9), and this manual delineation is time- and labor-consuming. Computer-assisted image autosegmentation can reduce the work stress of physicians while increasing the accuracy, consistency, and reproducibility of target region delineation. Therefore, it is especially important to use existing image segmentation techniques based on precise identification in the localization imaging of 3D BT for cervical cancer to develop automatic and accurate delineation software that is suitable for delineation of clinical target volumes (CTVs) and organs at risk (OARs) for 3D BT for cervical cancer.

In recent years, atlas-based automatic segmentation (ABAS) software $(10,11)$ has been frequently used in automatic segmentation of CTVs and OARs in radiotherapy. However, ABAS has its limitations. First, it is difficult to construct a "universal atlas" due to differences in patient age and the shape and size of organs. Second, image registration is an extremely time-consuming process due to its variability. Therefore, there is a need to use machine learning algorithms to solve the problem of CTV and OAR segmentation before radiotherapy. However, there are very few studies on the use of deep learning algorithms in CTV segmentation, especially in the BT treatment of cervical cancer. In contrast to OARs, CTVs do not have definite borders and include potential tumor dissemination or subclinical tissue regions that are almost undetectable in localization imaging. CTV segmentation depends largely on the physicians' professional knowledge.

In this paper, RefineNet (12) was used for automatic delineation of CTVs and OARs in the localization CT images for cervical cancer. Unlike existing image segmentation models, RefineNet introduces the multipath refinement network architecture to directly extract raw contextual information from the input images, and it employs remote residual connection to perform multiscale and multilayer feature learning and compensation of contextual features in high-level convolution layers. Therefore, RefineNet significantly increases precision compared with typical convolutional neural network segmentation models. In addition, this method is invariant to organ size, body shape, and age, as it can learn the relevant knowledge itself and successfully process input images that have large differences.

This study first employed CLAHE to preprocess input data to achieve image enhancement. Then, the RefineNet algorithm was used to construct automatic delineation models for the CTV and the OARs. Next, the models were cross-validated, and a clinical trial was carried out. In this way, rapid and accurate automatic delineation of the CTV 
and the OARs in 3D BT for cervical cancer was achieved. This shortens the waiting time of patients, decreases injury to normal tissues, decreases the incidence of complications, and greatly improves the working efficiency of medical staff, which will greatly benefit both patients and medical staff. We present the following article in accordance with the MDAR reporting checklist (available at https://dx.doi. org/10.21037/atm-21-4074).

\section{Methods}

\section{Experiment data}

In this study, 200 cervical cancer patients who underwent radical radiotherapy from January 2016 to December 2018 in our center were selected. The inclusion criteria were set through discussion with experts to obtain a high-quality expert database. The inclusion criteria were as follows: (I) first diagnosis of histopathologically confirmed cervical cancer and (II) no treatment of cervical cancer before enrollment. The expected number of patients was 200, and the number of enrolled patients was 200. The mean age was 57.8 years (32-83 years). Patients were pathologically confirmed to have squamous-cell carcinoma or adenocarcinoma at International Federation of Gynecology and Obstetrics (FIGO) stage [2009] IB-IVA. There were 9 stage IB2, 3 stage IIa1, 8 stage IIa2, 136 stage IIB, 16 stage IIIA, 22 stage IIIB, and 6 stage IVA cases. All patients underwent CT-guided 3D BT. This study was approved by the Institutional Review Board of our hospital.

The target regions were accurately delineated based on the 3D BT range for cervical cancer and gynecological examination results as recommended by GEC-ESTRO (4), and CT-based delineation was performed based on guidelines modified by Viswanathan et al. (13). After collecting the CT image data from patients, five radiologists used the Eclipse 11.0 system to delineate the high-risk CTV and OARs in 3D BT for cervical cancer. A radiotherapy expert with more than 10 years of experience was invited to manually adjust these delineated CTV and OARs, and the adjustment results were used as the gold standard. The delineation team and the radiology expert labeled the CT images and constructed a CTV and OAR image library. The mean duration of delineation of the CTV and OARs of a patient was $15-20$ minutes.

In this study, a universal multipath refinement network, the RefineNet-based deep learning protocol, was used to segment CTV and OARs in 3D BT for cervical cancer. In this study, $80 \%$ of cases in the database were included in the training set to train and adjust the model parameters, and the other $20 \%$ of cases were included in the validation set for assessing the model and for iteration and tuning. Finally, 10 patients who were treated in our center in 2019 were selected as the test cohort to generate automatically delineated contours for CTV and OARs.

\section{Data preprocessing}

Image enhancement: CLAHE was used for image enhancement, based on histogram equalization (HE) and adaptive histogram equalization (AHE), to improve the local contrast and increase the clarity of margins in every region in the image. This also prevented excessive noise amplification.

Figure 1 shows the image enhancement results of $\mathrm{HE}$, AHE, and CLAHE algorithms. The raw CT images had significantly better quality after image preprocessing, and there were great improvements in the shape and texture in image contents, including their border information.

\section{Experimental procedure and statistical analysis}

In this study, the TensorFlow system was used to carry out the proposed algorithms. The experiment was divided into the training and test stages. During the training stage, a cross-validation experiment was carried out, i.e., the dataset was randomly divided into five subsets of equal size. First, the model was trained using the first four subsets $(80 \%$ of data, i.e., CT images of 160 patients), and the model results were validated using the fifth subset $(20 \%$ of data, i.e., CT images of 40 patients). After that, another subset was selected as the validation set, and the other four subsets were used to train the model. The above steps were repeated until five models had been trained. To obtain better model results, we did not employ the pretraining strategy for initialization of network parameters in this study. Instead, the cervical cancer dataset of our center was directly used for model training. A stochastic gradient descent algorithm with a momentum of 0.99 was used to optimize the network parameters. In the test stage, the CT images of 10 cases were tested one by one, and the manual delineation of their CTVs and OARs was compared with the model's results to measure the performance of the model.

\section{Ethical statement}

All procedures performed in this study involving human 
Images
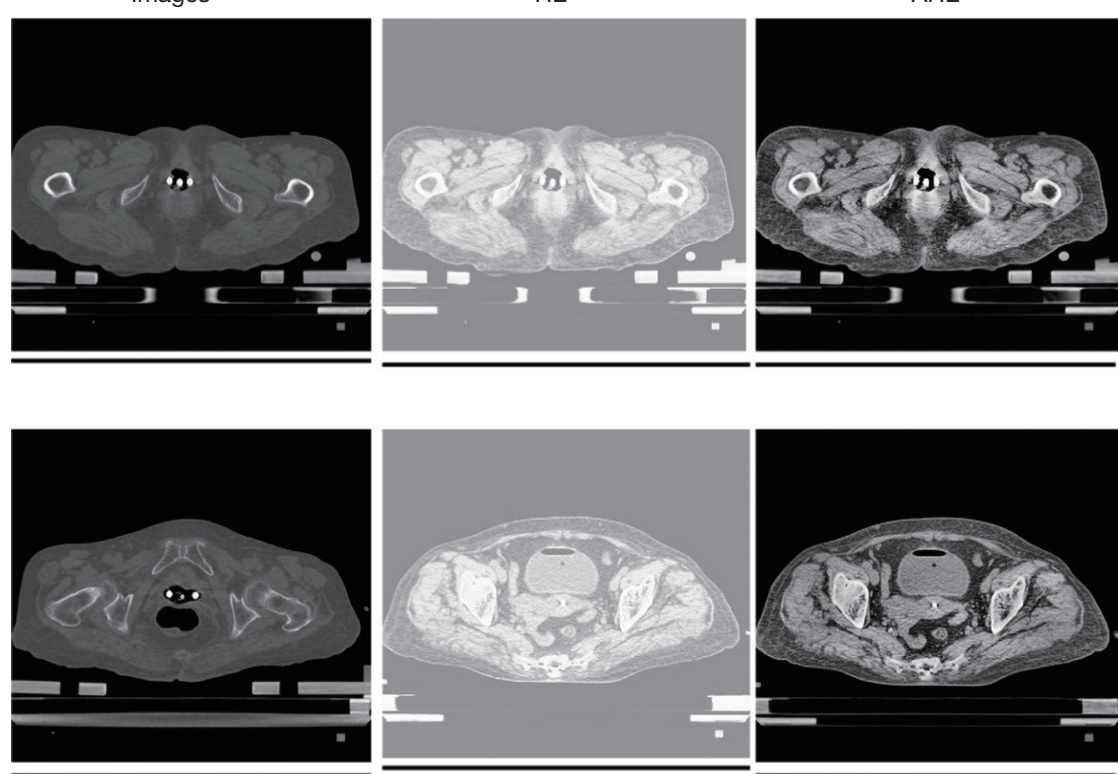

$\mathrm{AHE}$
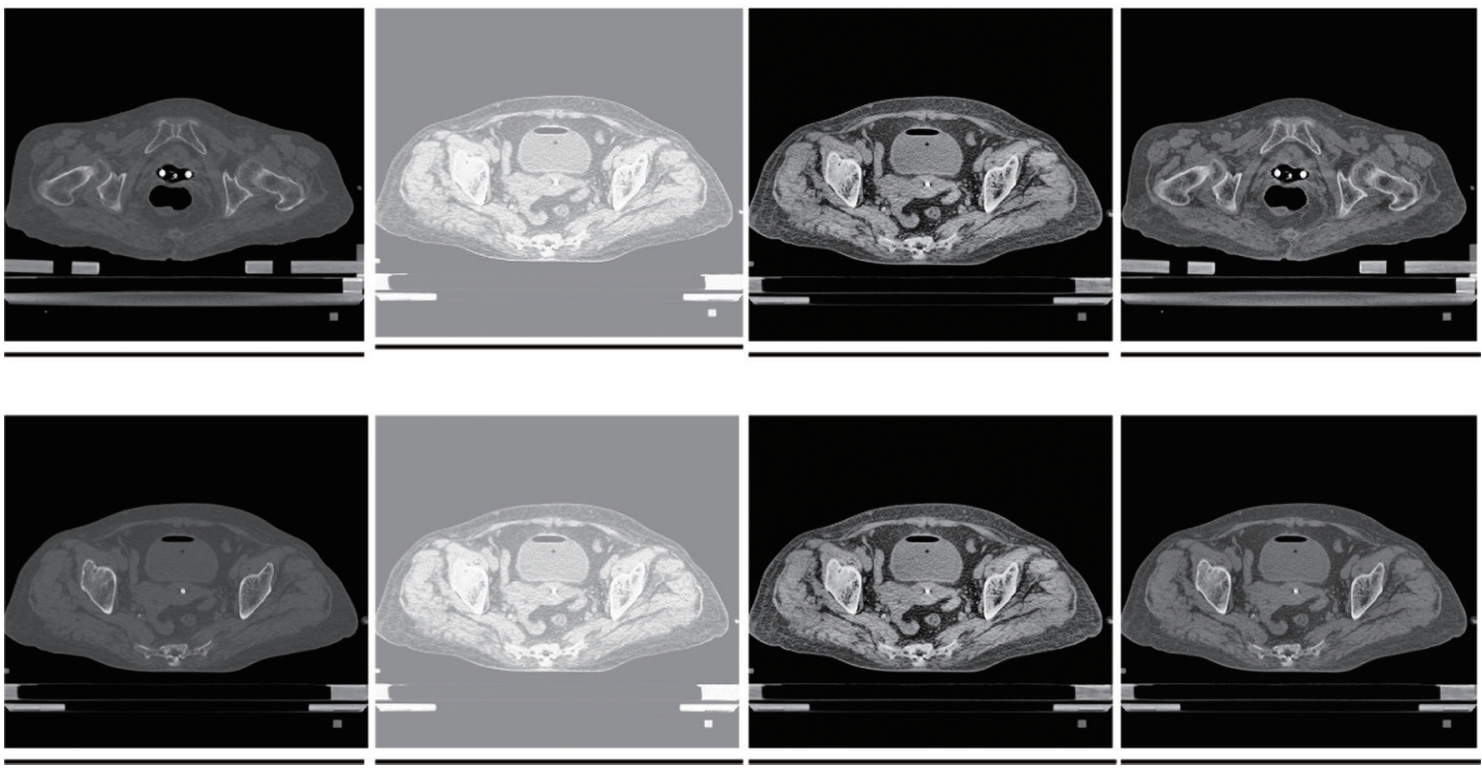
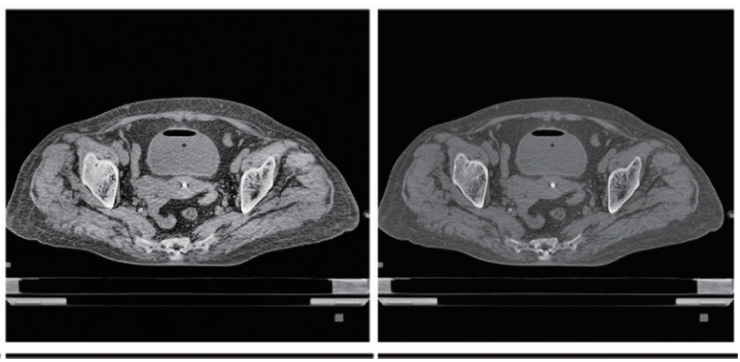

Figure 1 Image enhancement results of HE, AHE, and CLAHE algorithms. HE, histogram equalization; AHE, adaptive histogram equalization; CLAHE, contrast-limited adaptive histogram equalization.

participants were in accordance with the Declaration of Helsinki (as revised in 2013). This study was approved by the Institutional Review Board of our hospital and informed consent was taken from all patients.

\section{Results}

The Dice similarity coefficient (DSC), overlap index (OI), and Hausdorff distance (HD) were used as conformity parameters for the experimental results, and the corresponding durations of delineation were compared. DSC was defined as the percentage overlap between two target regions. OI is often used to measure the performance of network segmentation. HD is the relative distance between points with the greatest difference in two target regions.

\section{Cross-validation experimental results}

To obtain reliable and stable model results at the training stage of this study, 5-fold cross-validation experiments were conducted on the CTV and OAR datasets. As shown in Tables 1,2, the results of the five experiments were stable, and the model with the best results was ultimately selected for subsequent tests.

\section{Comparison of CTV and OAR volumes}

Volume scatter plots were used to show the volume results from automatic delineation of the CTV and OARs using the RefineNet-based model and using manual delineation. The overall distributions of the CTV from RefineNetbased automatic delineation and manual delineation were 
Table 1 Five-fold cross-validation experimental results of CTV

\begin{tabular}{|c|c|c|c|c|c|c|}
\hline Experiment number & \multicolumn{2}{|c|}{ DSC } & \multicolumn{2}{|c|}{ HD } & \multicolumn{2}{|c|}{ OI } \\
\hline First experiment & 0.832 & 0.073 & 9.589 & 4.076 & 0.826 & 0.142 \\
\hline Second experiment & 0.836 & 0.088 & 9.242 & 5.079 & 0.788 & 0.139 \\
\hline Third experiment & 0.796 & 0.270 & 9.525 & 5.034 & 0.769 & 0.271 \\
\hline Fifth experiment & 0.722 & 0.111 & 14.581 & 5.513 & 0.612 & 0.176 \\
\hline
\end{tabular}

CTV, clinical target volume; DSC, Dice similarity coefficient; HD, Hausdorff distance; OI, overlap index.

Table 2 Five-fold cross-validation experimental results of OARs

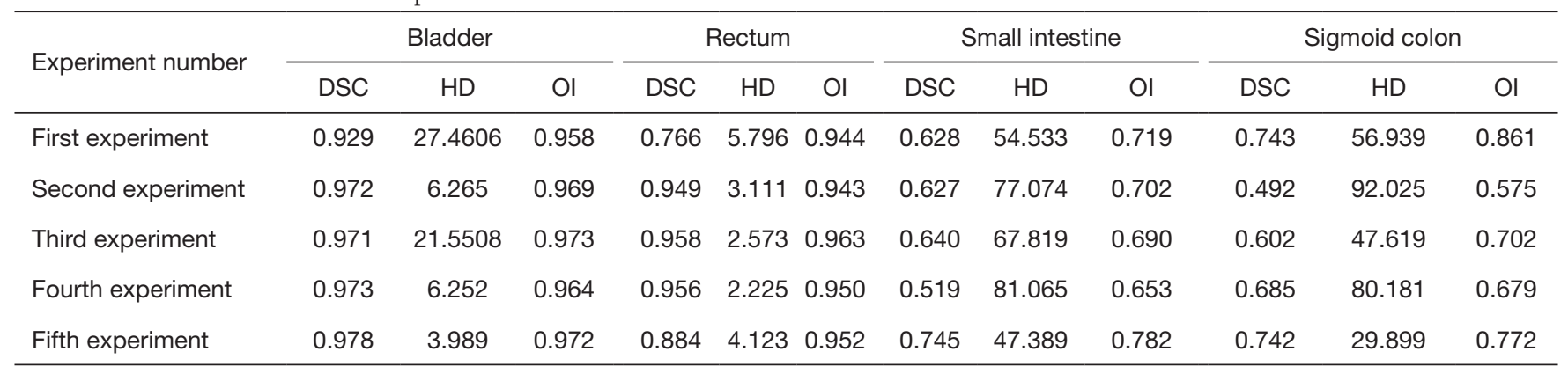

OAR, organ at risk; DSC, Dice similarity coefficient; HD, Hausdorff distance; OI, overlap index.

close to each other in the volume scatter plot (Figure 2). For the OARs, the volume scatter plots for the bladder and rectum were close (Figures 3,4), while the volume scatter plots for the small intestine and sigmoid colon differed greatly (Figures 5,6).

\section{Comparison of CTV and manual delineation}

In this study, the RefineNet-based model resulted in higher DSC, HD, and OI for segmented CTV (Table 3). As an intuitively display of the segmentation results of the RefineNet model, Figure 7 shows the automatic segmentation results for the CTV. The RefineNet-based automatic delineation results overlapped well with the manual delineation results.

\section{Comparison of automatically delineated and manually delineated OARs}

Likewise, automatically delineated OARs were compared with manually delineated OARs. In this study, the segmentation results for the bladder (DSC: $85.96 \%$ ) were the best among all the OAR segmentation results obtained by using the RefineNet-based model. This was mainly because the bladder has low contrast and a relatively regular shape. The segmentation result for the rectum (DSC: $85.83 \%$ ) was also good when compared with manual delineation. On the other hand, the qualities of the automatic delineation results for the sigmoid colon (DSC: $66.42 \%$ ) and small intestine (DSC: $56.32 \%$ ) were poorer than those of the other OARs. This was due to their complex shapes and variability (Table 4). Figure 8 intuitively displays the RefineNet model segmentation and manual delineation results. It can be clearly seen that the RefineNet delineation results highly overlapped with the manual delineation results in terms of both volume and shape.

\section{Time costs}

Next, RefineNet was trained. There were differences in training duration between all the models. The mean training duration for the CTV automatic delineation model was $112.8 \mathrm{~h}$, and the mean training durations for the four OAR automatic delineation models were 104, 86.2, 81.4, 

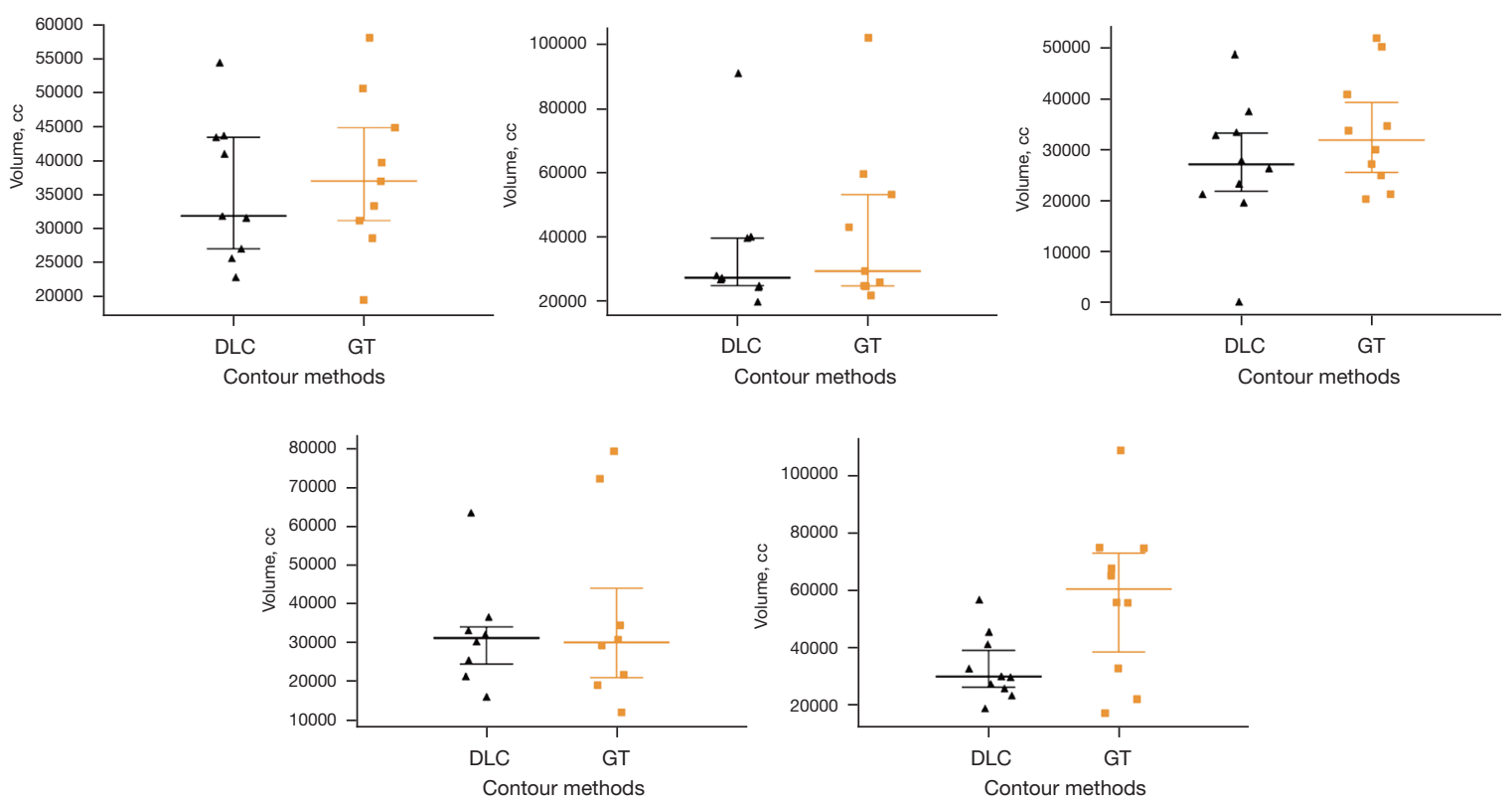

Figure 2 Volume scatter plot of RefineNet-based automatic delineation and manual delineation results of CTV. CTV, clinical target volume; DLC, automatically delineated range; GT, manually delineated range.
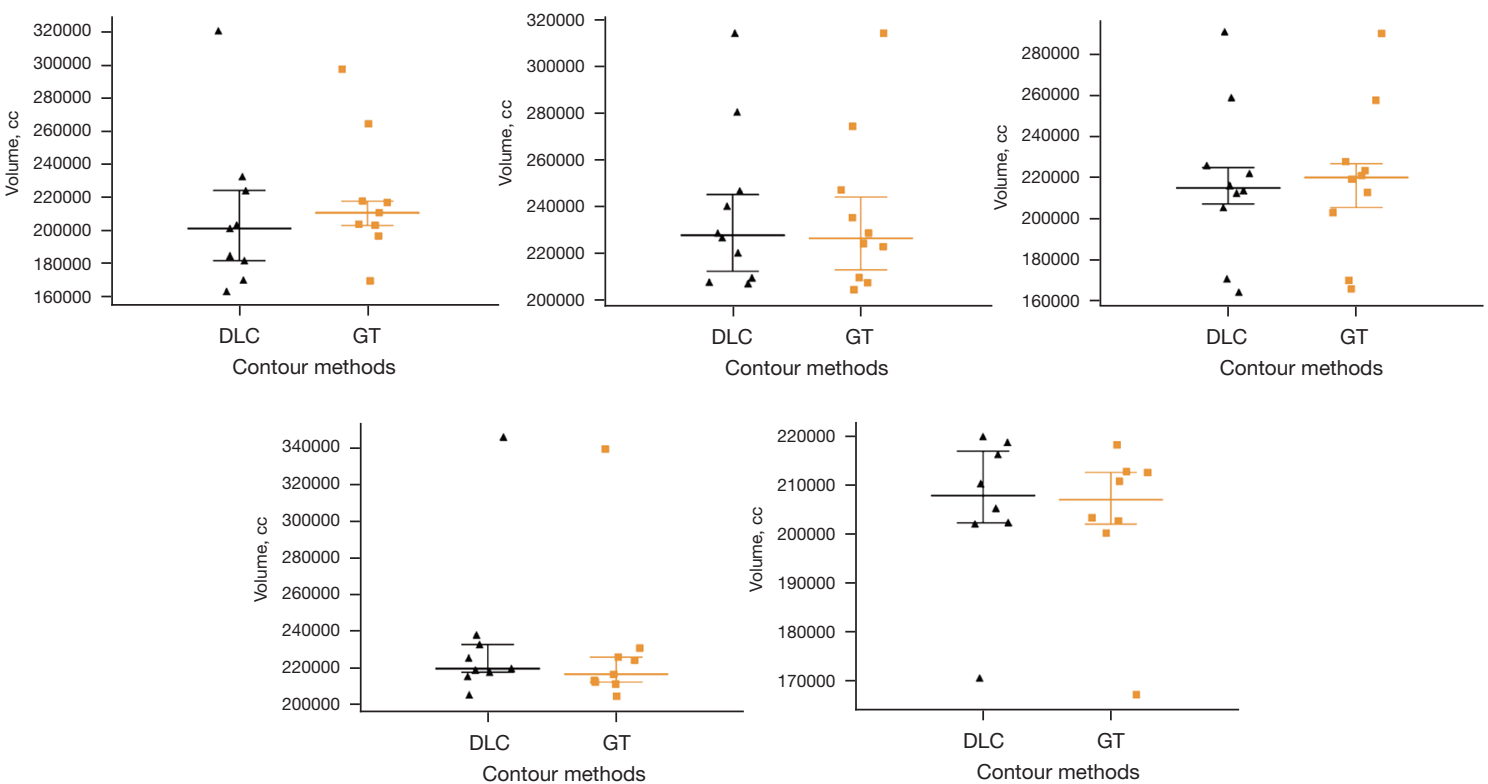

Figure 3 Volume scatter plot of RefineNet-based automatically delineated and manually delineated bladder. DLC, automatically delineated range; GT, manually delineated range.

and 82.2 h. The mean duration of RefineNet automatic contour processing of CTV was $70 \mathrm{~s}$, and the mean durations of automatic delineation of the bladder, rectum, sigmoid colon, and small intestine were $67,67.4,63.8$, and $60.8 \mathrm{~s}$, respectively. In our clinical center, the duration of manual delineation of CTVs and OARs by physicians is 15- 

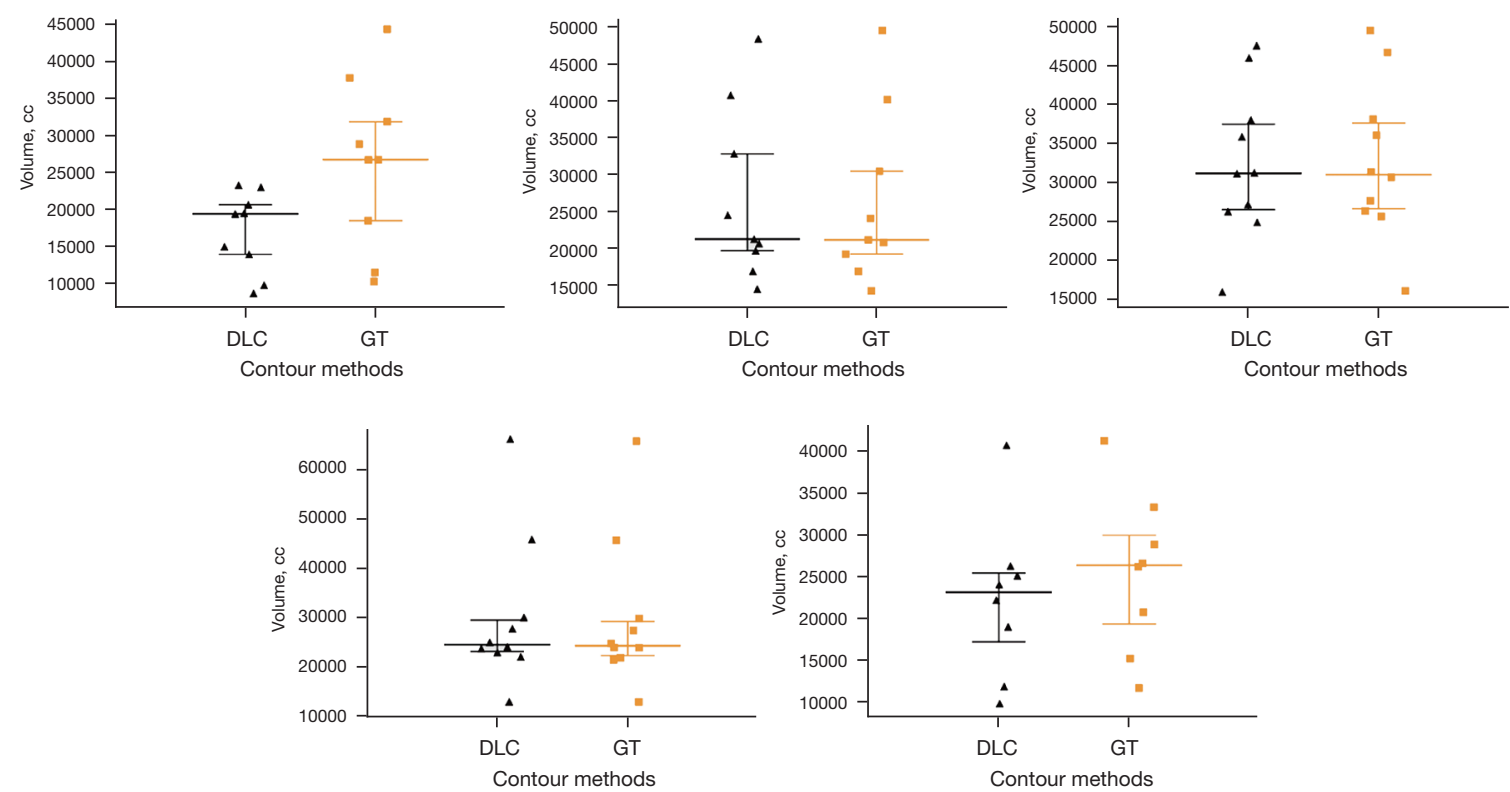

Figure 4 Volume scatter plot of RefineNet-based automatic delineation and manual delineation results of the rectum. DLC, automatically delineated range; GT, manually delineated range.
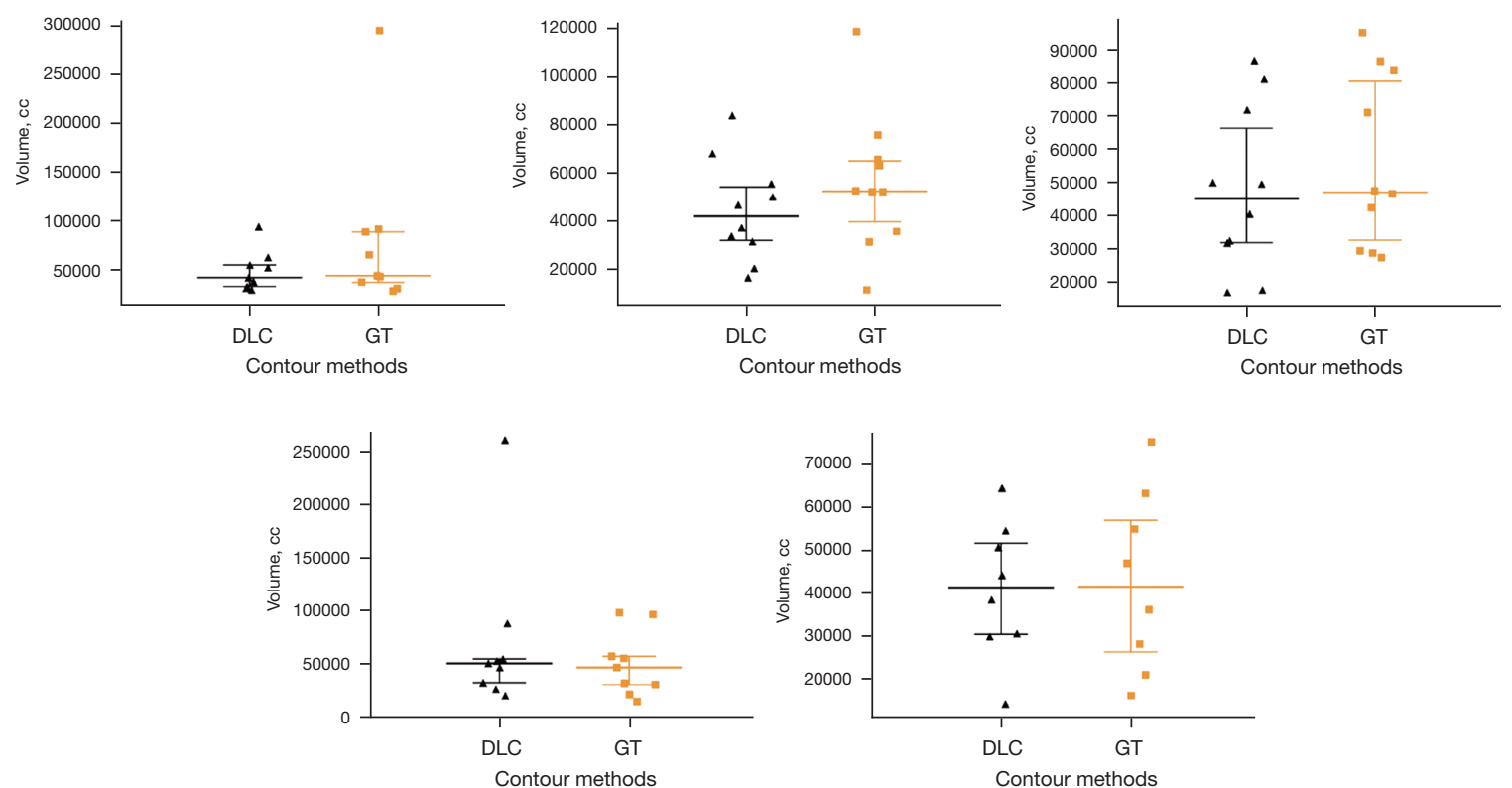

Figure 5 Volume scatter plot of RefineNet-based automatic delineation and manual delineation results of the sigmoid colon. DLC, automatically delineated range; GT, manually delineated range.

20 minutes. The total time saved by the RefineNet model was thus $60-75 \%$, which can greatly increase the work efficiency of medical staff and shorten the waiting time of patients.

\section{Discussion}

Cervical cancer is the most common reproductive system malignancy in women. Radiotherapy is suitable for patients 

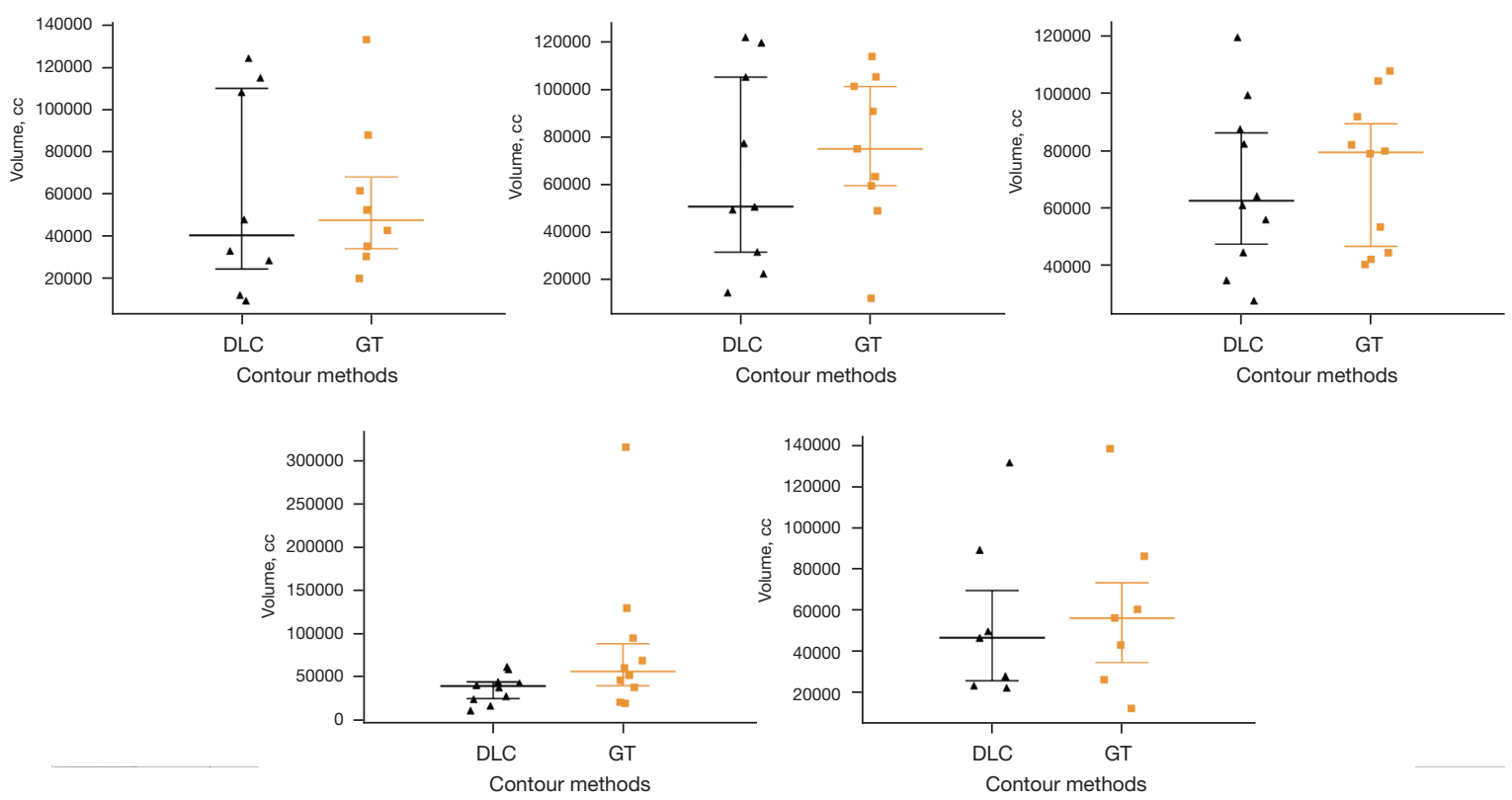

Figure 6 Volume scatter plot of RefineNet-based automatic delineation and manual delineation results of the small intestine. DLC, automatically delineated range; GT, manually delineated range.

Table 3 DSC, HD, and OI of RefineNet model-based CTV segmentation

\begin{tabular}{llllllllll}
\hline \multirow{2}{*}{ Model } & \multicolumn{2}{c}{ DSC } & & \multicolumn{2}{c}{ HD } & & \multicolumn{2}{c}{ Ol } \\
\cline { 2 - 3 } \cline { 7 - 8 } \cline { 7 - 8 } & Mean & Var & & Mean & Var & & Mean & Var \\
\hline RefineNet & 0.861 & 0.053 & & 6.005 & 4.048 & & 0.839 & 0.108 \\
\hline
\end{tabular}

DSC, Dice similarity coefficient; HD, Hausdorff distance; OI, overlap index; CTV, clinical target volume.

with all stages of cervical cancer, and intracavitary $\mathrm{BT}$ is an indispensable aspect of cervical cancer radiotherapy. The afterloading technique used in $3 \mathrm{D}$ BT is more accurate than conventional $3 \mathrm{D}$ radiotherapy and demonstrates the strengths of precision radiotherapy. 3D BT can achieve the best dose distribution in target regions, increase the dose to tumors, and reduce the dose to surrounding healthy tissues (3). At present, CT imaging is the most used guidance method for $3 \mathrm{D}$ BT, but it still has significant shortcomings in soft tissue resolution compared with MRI (13-17). Few institutions employ MRI to guide 3D BT. This study first employed CLAHE based on CT imaging guidance for preprocessing of input data to achieve image enhancement. Next, the RefineNet algorithm was used to construct automatic delineation models for the CTV and the OARs. The models were then cross-validated, and a clinical trial was carried out. In this way, rapid and accurate automatic delineation of the CTV and OARs for 3D BT for cervical cancer was achieved.

Precise and consistent delineation of tumor volumes and OARs is one of the most crucial steps in radiotherapy. However, delineation is a labor-intensive and timeconsuming step. Even though there are recommendations in well-accepted guidelines, CTV delineation quality is still largely determined by the expertise of each physician. There are large differences in clinical knowledge between physicians from different regions in China due to inequalities in facilities and manpower. Therefore, the introduction of a superior automatic delineation model would help improve the precision of target region delineation, decrease errors, and save time.

The individual experience and preferences of radiation oncologists will lead to deviations in target region delineation due to individual or external factors, and differences in target region delineation by different medical institutions and physicians are a source of uncertainty during radiotherapy $(17,18)$. Against the backdrop of precision radiotherapy, differences in target region delineation are becoming increasingly important as improvements in radiotherapy come from the minimization of errors in other areas (19). Leunens et al. (19) found that the interphysician and intraphysician variability in target region delineation was relatively high. Lu et al. (20) 


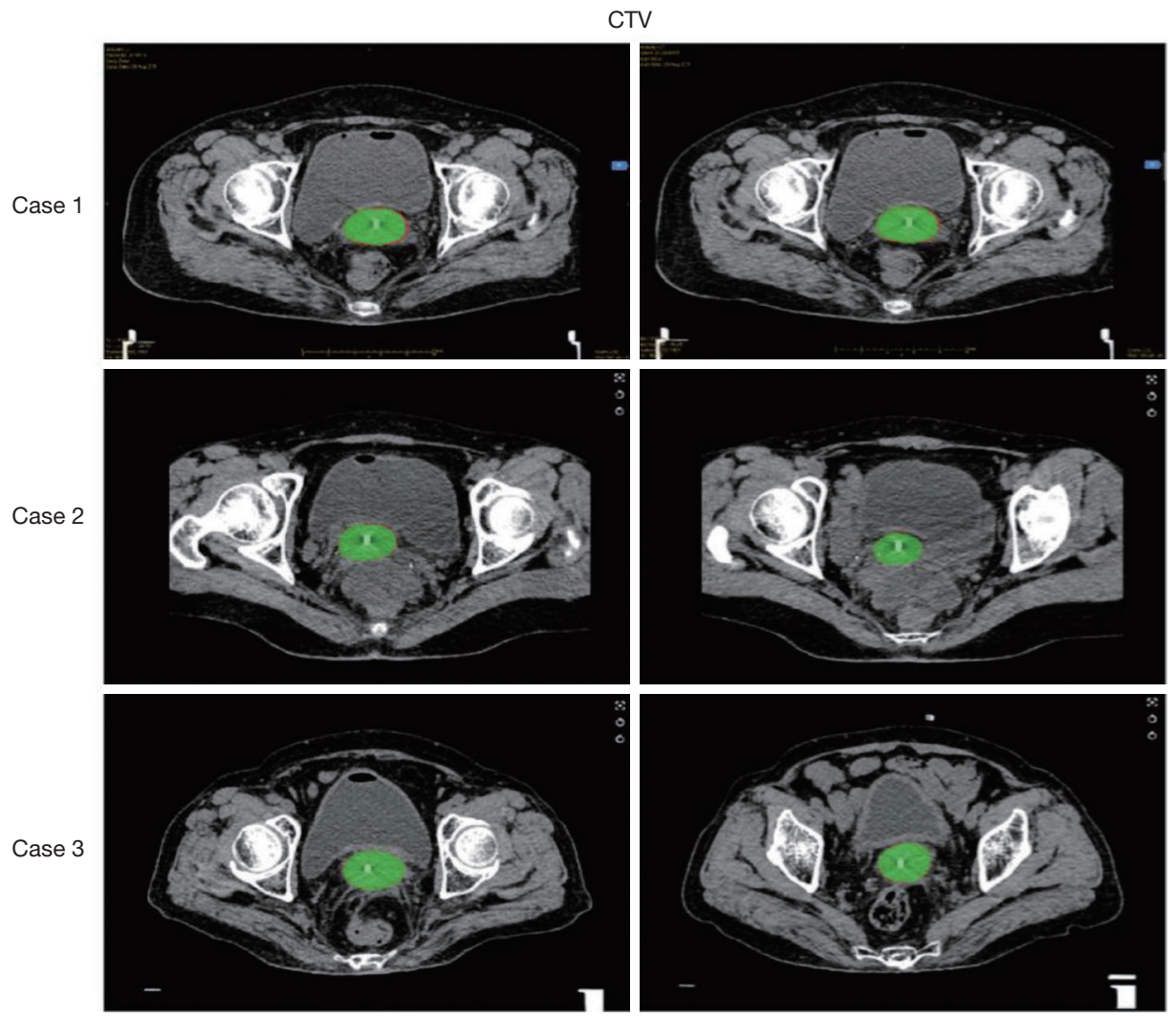

Figure 7 RefineNet model-based automatic delineation and manual delineation results of CTVs. Green-filled area: automatic delineation; red line: manual delineation. CTV, clinical target volume.

Table 4 DSC, HD, and OI of RefineNet model-based OAR segmentation

\begin{tabular}{|c|c|c|c|c|c|c|}
\hline OAR & \multicolumn{2}{|c|}{ DSC } & \multicolumn{2}{|c|}{$\mathrm{HD}$} & \multicolumn{2}{|c|}{ Ol } \\
\hline Bladder & 0.860 & 0.086 & 19.981 & 11.418 & 0.783 & 0.120 \\
\hline Rectum & 0.858 & 0.089 & 12.273 & 8.080 & 0.894 & 0.131 \\
\hline Sigmoid colon & 0.664 & 0.123 & 98.409 & 50.984 & 0.601 & 0.190 \\
\hline
\end{tabular}

DSC, Dice similarity coefficient; HD, Hausdorff distance; OI, overlap index; OAR, organ at risk.

reported differences in target region contours delineated by different radiation oncologists, and the DSC was only $75 \%$. Caravatta et al. (21) evaluated the overlap precision in CTVs delineated by different physicians and obtained a DSC of only $68 \%$. In recent years, advances have been made in computer-assisted automatic image segmentation. The consistency between target contours obtained from automatic delineation is higher than that between target contours obtained from manual delineation (22-25). Similar results showing outstanding target region consistency were obtained using the RefineNet-based model in this study. After cross-validation, a test experiment was carried out with comparison to manual delineation. The results further confirmed that the RefineNet-based model is an automatic delineation algorithmic model with high stability and consistency.

Another important problem in medical image segmentation is time consumption. The greatest strength 


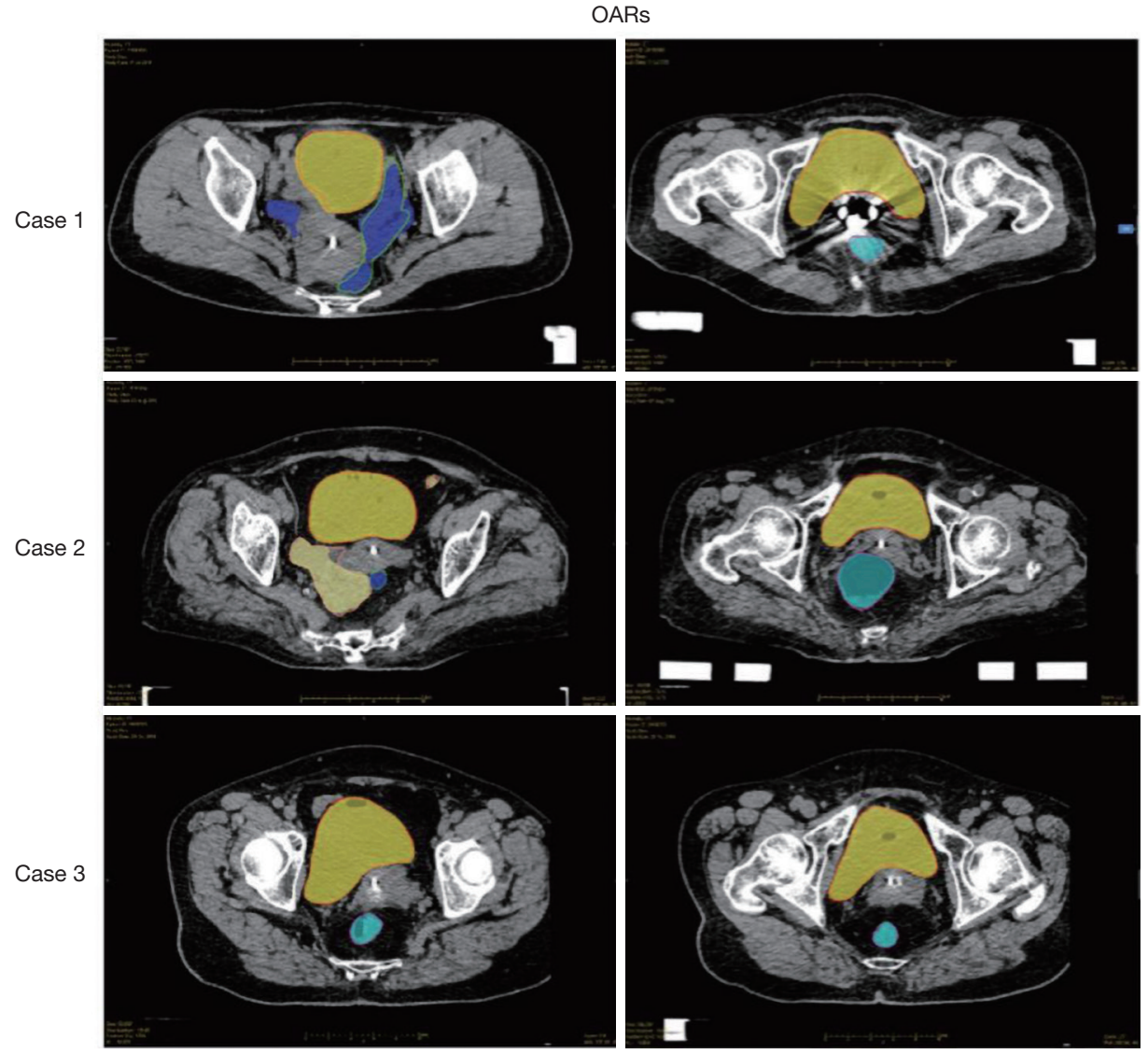

Figure 8 RefineNet model-based automatic delineation and manual delineation results of OARs. Filled area: automatic delineation; red line: manual delineation. OARs, organs at risk.

of automatic delineation is the direct provision of a possible contour solution that only needs to be adjusted by the physician, with no need for manual delineation of any contours. In this study, the RefineNet-based model took $60-70 \%$ less time than radiologists to delineate CTV contours. In practice, this could shorten the time that patients spend waiting for BT with indwelling catheters in their cervix or vaginal fornix and decrease the effects on visceral organs, the damage to healthy tissues, and the incidence of complications. At the same time, this method would increase the work efficiency of medical staff.

In this paper, a convolutional neural network deep learning-based protocol was used for segmentation of CTV and OARs in 3D BT for cervical cancer. The precision of automatic segmentation depends on the structure of the target lesion (26). As the CTV and OAR segmentations are carried out at the same time, it is not rational to directly compare the performance of this model with the performance of other software used for automatic segmentation of OARs only. The DSC values obtained in this paper were higher than the DSC values of clinical target regions in other studies. The segmentation results of the CTV or entire breast tissue in a recent publication were 0.78 (right breast) $/ 0.80$ (left breast), 0.89 (right breast) $/ 0.87$ (left breast) (26), and 0.87 (both breasts) (27). A publication by the Cancer Hospital at the Chinese Academy of Medical Sciences found that the DSC for the gross tumor volume, the gross tumor volume of lymph nodes, and the CTV obtained from automatic delineation of nasopharyngeal cancer were $80.9 \%, 62.3 \%$, and $82.6 \%$, respectively (28). In this study, the DSC values in 3D BT for high-risk cervical cancer were 0.861 for the CTV, 0.860 for the bladder, and 0.858 for the rectum.

This study shows that RefineNet can accurately segment CTV and OARs and perform better than U-Net. U-Net is one of the most popular methods in medical image analysis and was also the first deep learning algorithm used in radiotherapy. In U-Net, a transposed convolutional layer is 
deployed to connect the encoder layer to the decoder layer without compensating for raw texture information. U-Net is more sensitive to visible border regions but may lose some contextual information, resulting in U-Net-segmented regions being smaller than RefineNet-segmented regions (29). In contrast, RefineNet directly extracts multiscale contextual features from raw input images, which include abundant contextual information (such as borders, texture, and structural information). Therefore, the RefineNet model in this study yielded better DSC values than U-Net.

ABAS $(10,30,31)$ usually groups patients based on body shape or organ size (small, medium, and large) to train the corresponding model, and good results are obtained when the model is used for testing. However, Atlas-based methods cannot be widely used in patients of all body shapes. In contrast, good and comparable segmentation results were obtained in this study using the deep learning method proposed, with no need to construct different models for patients with different organ sizes or shapes and with the freedom to randomly select the training and test sets. Even with large differences in the input images, the method proposed in this paper can learn the information about these differences given enough examples, thereby generating excellent segmentation results.

The vast majority of early patients are cured by surgery and local advanced patients by concomitant radiotherapy and chemotherapy. However, for patients with recurrent and metastatic cervical cancer, the treatment effect is limited and the prognosis is very poor (32). In addition to the firstline treatment method of platinum based chemotherapy combined with bevacizumab, there is no particularly effective targeted treatment method (33). The effective rate of immunotherapy in the treatment of cervical cancer is low, so it can improve the cure rate of locally advanced cervical cancer. It is particularly important to reduce toxic and side effects. External irradiation radiotherapy and BT are one of the most important methods for the treatment of cervical cancer, so improving the treatment accuracy of radiotherapy is particularly important for the treatment of cervical cancer.

There are still some limitations to this study. In later studies, the sample size should be continuously expanded to increase the accuracy and robustness of the proposed models. In addition, different algorithms and models should be tested for statistical comparison, and external clinical data should be used for validation of the proposed models to give them greater clinical application value.

\section{Conclusions}

The RefineNet network has the potential to improve the consistency of target region delineation and simplify the operational procedures of radiotherapy. RefineNet can be used to construct automatic delineation models to achieve rapid, accurate, automatic delineation of 3D BT CTVs and OARs for cervical cancer. This method shortens the waiting time of patients and improves the work efficiency of medical staff.

\section{Acknowledgments}

Funding: This study was supported by a grant from the Natural Science Foundation of Zhejiang Province (grant number: LQ19H160032).

\section{Footnote}

Reporting Checklist: The authors have completed the MDAR reporting checklist. Available at https://dx.doi. org/10.21037/atm-21-4074

Data Sharing Statement: Available at https://dx.doi. org/10.21037/atm-21-4074

Conflicts of Interest: All authors have completed the ICMJE uniform disclosure form (available at https://dx.doi. org/10.21037/atm-21-4074). The authors have no conflicts of interest to declare.

Ethical Statement: The authors are accountable for all aspects of the work in ensuring that questions related to the accuracy or integrity of any part of the work are appropriately investigated and resolved. All procedures performed in this study involving human participants were in accordance with the Declaration of Helsinki (as revised in 2013). This study was approved by the Institutional Review Board of our hospital and informed consent was taken from all patients.

Open Access Statement: This is an Open Access article distributed in accordance with the Creative Commons Attribution-NonCommercial-NoDerivs 4.0 International License (CC BY-NC-ND 4.0), which permits the noncommercial replication and distribution of the article with the strict proviso that no changes or edits are made and the original work is properly cited (including links to both the 
formal publication through the relevant DOI and the license). See: https://creativecommons.org/licenses/by-nc-nd/4.0/.

\section{References}

1. Chen W, Sun K, Zheng R, et al. Cancer incidence and mortality in China, 2014. Chin J Cancer Res 2018;30:1-12.

2. Liang $\mathrm{B}$, Yu H, Huang $\mathrm{L}$, et al. A prognostic model guides surgical resection in cervical squamous cell carcinoma. Transl Cancer Res 2020;9:1711-31.

3. Pötter R, Dimopoulos J, Georg P, et al. Clinical impact of MRI assisted dose volume adaptation and dose escalation in brachytherapy of locally advanced cervix cancer. Radiother Oncol 2007;83:148-55.

4. Haie-Meder C, Pötter R, Van Limbergen E, et al. Recommendations from Gynaecological (GYN) GECESTRO Working Group (I): concepts and terms in 3D image based 3D treatment planning in cervix cancer brachytherapy with emphasis on MRI assessment of GTV and CTV. Radiother Oncol 2005;74:235-45.

5. Pizer SM, Johnston RE, Ericksen JP, et al. Contrastlimited adaptive histogram equalization: speed and effectiveness. Proceedings of the First Conference on Visualization in Biomedical Computing 1990:337-45.

6. Geets X, Daisne JF, Arcangeli S, et al. Inter-observer variability in the delineation of pharyngo-laryngeal tumor, parotid glands and cervical spinal cord: comparison between CT-scan and MRI. Radiother Oncol 2005;77:25-31.

7. Kepka L, Bujko K, Garmol D, et al. Delineation variation of lymph node stations for treatment planning in lung cancer radiotherapy. Radiother Oncol 2007;85:450-5.

8. Pizer SM, Amburn EP, Austin JD, et al. Adaptive histogram equalization and its variations. Computer Vision, Graphics, and Image Processing 1987;39:355-68.

9. Wang F, Tang Q, Lv G, et al. Comparison of computed tomography and magnetic resonance imaging in cervical cancer brachytherapy: A systematic review. Brachytherapy 2017;16:353-65.

10. Lee LK, Liew SC, Thong WJ. A review of image segmentation methodologies in medical image. In: Sulaiman HA, Othman MA, Othman MFI, et al. editors. Advanced Computer and Communication Engineering Technology. Cham: Springer, 2015;315:1069-80.

11. Lin A, Kubicek G, Piper JW, et al. Atlas-based segmentation in prostate IMRT: Timesavings in the clinical workflow. Int J Radiat Oncol Biol Phys 2008;72:S328-9.
12. Lin G, Milan A, Shen C, et al. RefineNet: Multi-path refinement networks for high-resolution semantic segmentation. In: Proceedings of the IEEE conference on computer vision and pattern recognition, 2017:1925-34.

13. Viswanathan AN, Dimopoulos J, Kirisits C, et al. Computed tomography versus magnetic resonance imaging-based contouring in cervical cancer brachytherapy: results of a prospective trial and preliminary guidelines for standardized contours. Int J Radiat Oncol Biol Phys 2007;68:491-8.

14. Hricak H, Gatsonis C, Coakley FV, et al. Early invasive cervical cancer: CT and MR imaging in preoperative evaluation - ACRIN/GOG comparative study of diagnostic performance and interobserver variability. Radiology 2007;245:491-8.

15. Tuntipumiamorn L, Lohasammakul S, Dankulchai P, et al. Comparison of impact of target delineation of computed tomography- and magnetic resonance imaging-guided brachytherapy on dose distribution in cervical cancer. J Contemp Brachytherapy 2018;10:418-24.

16. Pötter R, Federico M, Sturdza A, et al. Value of Magnetic Resonance Imaging Without or With Applicator in Place for Target Definition in Cervix Cancer Brachytherapy. Int J Radiat Oncol Biol Phys 2016;94:588-97.

17. Vinod SK, Jameson MG, Min M, et al. Uncertainties in volume delineation in radiation oncology: A systematic review and recommendations for future studies. Radiother Oncol 2016;121:169-79.

18. Louie AV, Rodrigues G, Olsthoorn J, et al. Inter-observer and intra-observer reliability for lung cancer target volume delineation in the 4D-CT era. Radiother Oncol 2010;95:166-71.

19. Leunens G, Menten J, Weltens C, et al. Quality assessment of medical decision making in radiation oncology: variability in target volume delineation for brain tumours. Radiother Oncol 1993;29:169-75.

20. Lu L, Cuttino L, Barani I, et al. SU-FF-J-85: InterObserver Variation In The Planning Of Head/Neck Radiotherapy. Med Phys 2006;33:2040.

21. Caravatta L, Macchia G, Mattiucci GC, et al. Interobserver variability of clinical target volume delineation in radiotherapy treatment of pancreatic cancer: a multiinstitutional contouring experience. Radiat Oncol 2014;9:198.

22. Lin L, Dou Q, Jin YM, et al. Deep Learning for Automated Contouring of Primary Tumor Volumes by MRI for Nasopharyngeal Carcinoma. Radiology 2019;291:677-86. 
23. Pallavaram S, D'Haese PF, Lake W, et al. Fully automated targeting using nonrigid image registration matches accuracy and exceeds precision of best manual approaches to subthalamic deep brain stimulation targeting in Parkinson disease. Neurosurgery 2015;76:756-65.

24. Nouranian S, Mahdavi SS, Spadinger I, et al. A multi-atlasbased segmentation framework for prostate brachytherapy. IEEE Trans Med Imaging 2015;34:950-61.

25. van Baardwijk A, Bosmans G, Boersma L, et al. PETCT-based auto-contouring in non-small-cell lung cancer correlates with pathology and reduces interobserver variability in the delineation of the primary tumor and involved nodal volumes. Int J Radiat Oncol Biol Phys 2007;68:771-8.

26. Sharp G, Fritscher KD, Pekar V, et al. Vision 20/20: perspectives on automated image segmentation for radiotherapy. Med Phys 2014;41:050902.

27. Anders LC, Stieler F, Siebenlist K, et al. Performance of an atlas-based autosegmentation software for delineation of target volumes for radiotherapy of breast and anorectal cancer. Radiother Oncol 2012;102:68-73.

28. Men K, Chen X, Zhang Y, et al. Deep Deconvolutional Neural Network for Target Segmentation of Nasopharyngeal Cancer in Planning Computed Tomography Images. Front Oncol 2017;7:315.

Cite this article as: Jiang X, Wang F, Chen Y, Yan S. RefineNet-based automatic delineation of the clinical target volume and organs at risk for three-dimensional brachytherapy for cervical cancer. Ann Transl Med 2021;9(23):1721. doi: 10.21037/atm-21-4074
29. Ronneberger O, Fischer P, Brox T. U-Net: Convolutional Networks for Biomedical Image Segmentation. In: Navab N, Hornegger J, Wells W, et al. editors. International Conference on Medical image computing and computerassisted intervention. Cham: Springer, 2015:234-41.

30. Steenbergen P, Haustermans K, Lerut E, et al. Prostate tumor delineation using multiparametric magnetic resonance imaging: Inter-observer variability and pathology validation. Radiother Oncol 2015;115:186-90.

31. $\mathrm{Hu} \mathrm{K}$, Lin A, Young A, et al. Timesavings for contour generation in head and neck IMRT: Multi-institutional experience with an atlas-based segmentation method. Int J Radiat Oncol Biol Phys 2008;72:S391.

32. Rosen VM, Guerra I, McCormack M, et al. Systematic Review and Network Meta-Analysis of Bevacizumab Plus First-Line Topotecan-Paclitaxel or Cisplatin-Paclitaxel Versus Non-Bevacizumab-Containing Therapies in Persistent, Recurrent, or Metastatic Cervical Cancer. Int J Gynecol Cancer 2017;27:1237-46.

33. Tewari KS, Sill MW, Penson RT, et al. Bevacizumab for advanced cervical cancer: final overall survival and adverse event analysis of a randomised, controlled, open-label, phase 3 trial (Gynecologic Oncology Group 240). Lancet 2017;390:1654-63. 